\title{
Autorenverzeichnis 1991
}

Agternkamp, C, 301

Bäßler, K.H., 5 Bauer, K.H., 233 Becker, A., 292 Behrendt, W, 20, 271 Bein, N., 233 Bergmann, H., 12 Biesel, E., 306 Böck, M., 30,137 Böhles, H., 224 Böhrer, H., 96 Burger, J., suppl 1:13

Canzler, H., 80 Carpentier, Y.A., 167

DAttelis, N.P., 167 Dahlan, W, 167 Korte, de, D., suppl 1:10 Dekker,W.J.A., suppl 1:10

Diehm,H., 137 Dietrich, G., 196 Dobler, G., 248 Doehn, M, 297 Drescher, M., 248

Eisenhardt, G., suppll: 24 Elwyn, D.H., 167 Fauth, U., 213 Förster, H., 236

Fürst, P., 167 Fusch, C, 85

Giani, G., 20 Gmür, J., suppl 1:13 Greither, L, 30,137 Gudden, A., 30,137 Gïidemann, C, 143

Halmágyi, M., 213 Haslinger-Matzenauer,

M.,114 Heilmann, L., 236 Heim, M.U., 137 Heine, W., 160 Himmelsbach, S., 30 Hofmann, M., 227 Hofmann-Preiß, K.,

292 Hojinackị, B., 236 Holtmann, M., 244 Homann, H.-H., 233 Hundelshausen, v., B.,

248

Iffland, H., 123

Jerke, A.S., 109

Kaniecki, K., 306 Karger, H., 114

Kemen, M., 26,199, 233 Kerbl,H., 114 Kirchdorfer, K., 114 Kleemann, P.P., 227 Klein, H.G., suppl 1:31 Klosterhalfen, B., 301 Kretschmer, V, 188,196,

suppl 1: 24 Krier, C., 96 Krützfeldt, A., 244 Kuchenbecker, 285

Lauermann, I., 130 Lerut, J., 91 Liebe, v., S., 26 Löhlein, D., 182 Löhmer, P., 301 Lorch, E., 236

Lothaller, M.-A., 280 Luboldt, W, 244

Mempel, W, 30,137 Mengelkamp, A.K., 244 Mertens, H., 301 Meyer, H.J., 80 Michalk, D., 224

Moeller, H., 85 Moog, R., 244 Müller, E., suppl 1:13 Müller, EG., 301 Müller, J.M., 109,123

Müller, M.J., 80 Müller, N., suppl 1: 19 Müller, P., 91 Mumme, A., 199 Müntefering, H., 236

Murphy, S., suppl 1: 3

Nathrath, M., 248 Nydegger, U., 91

Oelz,O., suppl 1:13

Paar, D., 244 Petcold, L., 114 Pflüger,H.H.,196 Pietersz, R.N.I., suppl 1:10 Planitz, C, 196

Rabast, U., 172 Raumanns, J., 20 Reesink, H.W., suppl 1:10 Reichlin, M., suppl 1:13 Reissigl,

H., 3 Richelle, M., 167 Rosmann van Goethem,

J., 114 Rossa, W., suppl 1: 24 Rössle, C., 167

Sailer, S., 292 Schanz, U., suppl 1:13

Schneck, H.J., 248 Schulze, C.G., 297 Selberg, O., 80 Simon, H.B., 301 Sperling, H., 199

Staehler, G., 143 Stehle, P., 167 Surmann, M., 20, 271

Uhrdahl, S.G., suppl 1:29

Weimann, A., 80 Wendt-Göknur, v., E., 224 Widhalm, K., 280 Wiesel, M, 143 Wölfelschneider, K., 123 Wolters, U., 109,123 
Zadravec, S., 114 Zhang, Z.H., 96 Zielke, E., 306 Zimmerli, M., 91 Zimmermann, A., 91 Zumtobel, W, 26,199, 233

(Autoren von Abstracts sind nicht im Jahres-gesamtverzeichnis auf-geführt) 